\title{
On the Minimal Adequate Sampling Frequency of the Photoplethysmogram for Pulse Rate Monitoring and Heart Rate Variability Analysis in Mobile and Wearable Technology
}

\author{
Szabolcs Béres, Lörinc Holczer, László Hejjel \\ Heart Institute, Medical Faculty, University of Pécs, Ifjúság str., 13, H-7624, Pécs, Hungary, hejjel.laszlo@pte.hu
}

\begin{abstract}
Recently there has been great interest in photoplethysmogram signal processing. However, its minimally necessary sampling frequency for accurate heart rate variability parameters is ambiguous. In the present paper frequency-modulated $1.067 \mathrm{~Hz}$ cosine wave modelled the variable PPG in silico. The five-minute-long, $1 \mathrm{~ms}$ resolution master-signals were decimated (D) at 2-500 ms, then cubic spline interpolated (I) back to $1 \mathrm{~ms}$ resolution. The mean pulse rate, standard deviation, root mean square of successive pulse rate differences (RMSSD), and spectral components were computed by Varian 2.3 and compared to the master-series via relative accuracy error. Also Poincaré-plot morphology was assessed. Mean pulse rate is accurate down to $303 \mathrm{~ms}$ (D) and $400 \mathrm{~ms}$ (I). In low-variability series standard deviation required at least $5 \mathrm{~ms}$ (D) and $100 \mathrm{~ms}$ (I). RMSSD needed $10 \mathrm{~ms}$ (D), and $303 \mathrm{~ms}$ (I) in normal, whereas $2 \mathrm{~ms}$ (D) and $100 \mathrm{~ms}$ (I) in lowvariability series. In the frequency domain $5 \mathrm{~ms}$ (D) and $100 \mathrm{~ms}$ (I) are required. $2 \mathrm{~ms}$ (D) and $100 \mathrm{~ms}$ (I) preserved the Poincaré-plot morphology. The minimal sampling frequency of PPG for accurate HRV analysis is higher than expected from the signal bandwidth and sampling theorem. Interpolation improves accuracy. The ratio of sampling error and expected variability should be considered besides the inherent sensitivity of the given parameter, the interpolation technique, and the pulse rate detection method.
\end{abstract}

Keywords: Photoplethysmography, heart rate variability, sampling frequency, spline interpolation, wearable health.

\section{INTRODUCTION}

Due to health professional and public expectations, wearable technology is being integrated into our everyday life facilitated by the rapid advances in smart devices, material sciences, sensor technology, and wireless communication [1]-[3]. The potential target populations are patients with chronic diseases, the elderly, professional sportsmen, highrisk personnel (soldier, policeman, fireman, etc.), and the general population during activities promoting good health. Since the beginning [4]-[6] these monitoring devices have gone through a fast evolution resulting in smaller, more intelligent, and more reliable gadgets, mostly commercially available by mass producers like the Polar watch [7]-[9], and a myriad of other newer wrist-worn devices [10]-[12].

The challenges coming with acquiring an ECG signal during activity (uncomfortable chest belt, separate ECG transmitter or long patient cables, assuring good galvanic contact or using capacitive electrodes with their own drawbacks) turned the interest towards optoelectronic or else photoplethysmographic (PPG) sensors. PPG signal can be as reliable as ECG for heart rate measurement and heart rate variability (HRV) analysis [13], [14]. Complete PPG chips, including integrated analogue front-end, $\mathrm{AD}$-converter, controller unit and serial communication module with or without incorporated LEDs and photosensor, are readily available on the market. These chips - thanks to their miniaturization, low power consumption and reliability - are embedded into the above mentioned wearables. Acquiring PPG signal on the wrist by a smartwatch avoids the use of a chest trap and a separate ECG transmitter unit. However, PPG registration also has its own technical problems [9], [11], [14].

Recently, instantaneous pulse rate [15] and HRV [16] were extracted accurately with special software from the PPG signal acquired by touching the smartphone's camera and flash with a fingertip. On the contrary, Bouts et al. [9] found iOS-based heart rate apps inferior to standard ECG or Polar heart rate monitor during exercise. Furthermore, a commercial webcam with appropriate software can be capable of extracting the PPG signal and pulse rate from the face of a person without skin contact [17], [18]; the dynamically spreading method is called "remote PPG" and can be used in real time or in recorded videos as well.

HRV analysis captures the delicate beat-to-beat heart rate fluctuations; hence the accurate instantaneous heart rate detection is crucial. Digitization results in a sampling error via transforming the continuous analogue signal into an 
impulse train with certain temporal resolution [19]. This error can be comparable in magnitude to the physiological jitter of the heart rate interval causing invalid measures. So the ratio of intrinsic variability and the sampling error could not be ignored during time domain [20], frequency domain [21], or non-linear [22] analysis. However, Ziemssen et al. [23] established just a minor difference in $100 \mathrm{~Hz}$ or $200 \mathrm{~Hz}$ versus the $500 \mathrm{~Hz}$ sampling during spectral analysis. Singh et al. [22] found significant error of sample entropy calculation at 125 or $250 \mathrm{~Hz}$ compared to $2000 \mathrm{~Hz}$ sampling frequency which was influenced by the length of the record as well. Some decades ago HRV analysis was mostly based on Holter monitors which were available at that time only with low sampling frequency $(100-250 \mathrm{~Hz})$. This stimulated the investigation of different interpolation techniques in order to improve the temporal resolution of the ECG and thus the accuracy of HRV estimates [24].

Nowadays $1000 \mathrm{~Hz}$ or higher sampling rate is easily available for analytical instrumentation; however, the lowest possible sampling frequency of biological signals came to the front again in the era of smart devices and wearables in order to minimize power consumption, memory needs and transmission bandwidth. Thus, interpolation methods considering computational needs and also power consumption - are investigated again for accurate HRV analysis. Baek et al. [25] found as low as $20 \mathrm{~Hz}$ sampling of the PPG signal sufficient to "assess trends" in HRV after interpolation; however, the reference values cannot be reproduced exactly. Mahdiani et al. [26] demonstrated accurate time domain parameters with cubic interpolation down to $50 \mathrm{~Hz}$ resampling of the original $5 \mathrm{kHz}$-sampled ECG. Choi and Shin [27] demonstrated particularly low sampling frequency of $25 \mathrm{~Hz}$ without interpolation for PPG signal acquisition.

The minimal adequate sampling for pulse rate calculation and especially for HRV analysis is still indecisive because of its multifactorial nature: it may depend on the intrinsic variability of the proband (healthy or with reduced variability [20]), the possible interpolation method (if any), other preprocessing of the signal (digital filtering, smoothing, derivation), the pulse rate detection method (curve fitting methods act like interpolation, but not so the single-point based methods, e.g., threshold techniques), and also the analyzed HRV parameters can show different inherent sensitivity to the sampling error (e.g., mean heart rate versus RMSSD). Present paper aims to find the minimally sufficient sampling frequency of the PPG signal for the time domain, frequency domain, and visual Poincaré-plot analysis without and with interpolation.

\section{METHODS}

2.1. Synthesis of photoplethysmograms, decimation and interpolation process

In order to control the spectral parameters and avoid artefacts, the PPG signal was generated on a PC as a sinusoidally narrow-band frequency-modulated cosine wave imitating pulse waves with sinus arrhythmia in normal and seriously reduced variability series. The last feature was determined by the maximal frequency drift from the carrier frequency.

The four records of five-minute long [28] PPG signals were modelled as a frequency modulated cosine wave with $1 \mathrm{~ms}$ temporal and 2001 points vertical resolution (codomain: $0 . .2000$ arbitrary units) in OriginPro 2017 software (OriginLab Corp., MA). The mean pulse rate interval (PPI) was chosen to be $937 \mathrm{~ms}$ equivalent to $1.067 \mathrm{~Hz}$ carrier frequency or 64.03 beat/minute in all four master PPGs (1). The sinusoidal modulation frequency $\left(f_{\text {mod }}\right)$ was $0.23 \mathrm{~Hz}$ mimicking the respiratory sinus arrhythmia at 13.8 breath/minute or $4347.8 \mathrm{~ms}$ period time in two series (2-3) and $0.11 \mathrm{~Hz}$ in the other two series (4-5) simulating the low frequency band HRV (9090.9 ms period time). Above integer base values are prime numbers in order to minimize synchronization within the signal. The maximum frequency deviation $\left(f_{\text {dev }}\right)$ was set to $0.05 \mathrm{~Hz}$ in one pair $(2,4)$ and $0.01 \mathrm{~Hz}$ in the other pair $(3,5)$ of series imitating normal and seriously reduced variability signals, respectively. The synthesized master PPG record with $1 \mathrm{~ms}$ resolution was decimated at 2, 5, 10, 20, 50, 100, 200, 303, 350, 400, and $500 \mathrm{~ms}$ and then each of them was cubic spline interpolated back to $1 \mathrm{~ms}$ resolution in OriginPro. Fig.1. shows the Fourier analysis of the master signal with $0.05 \mathrm{~Hz}$ frequency deviation and the location of the higher decimation values elucidating the reason of their particular choice.

$$
\begin{gathered}
P P I[t]=A+A \times \cos \left[\frac{2 \pi}{P P_{\text {mean }}} t+\frac{f_{\text {dev }}}{f_{\text {mod }}} \sin \left(2 \pi \times \frac{f_{\text {mod }}}{1000} t\right)\right] \\
P P I[t]=1000+1000 \times \cos \left[\frac{2 \pi}{937} t+\frac{0.05}{0.23} \sin \left(2 \pi \times \frac{0.23}{1000} t\right)\right] \\
P P I[t]=1000+1000 \times \cos \left[\frac{2 \pi}{937} t+\frac{0.01}{0.23} \sin \left(2 \pi \times \frac{0.23}{1000} t\right)\right] \\
P P I[t]=1000+1000 \times \cos \left[\frac{2 \pi}{937} t+\frac{0.05}{0.11} \sin \left(2 \pi \times \frac{0.11}{1000} t\right)\right] \\
P P I[t]=1000+1000 \times \cos \left[\frac{2 \pi}{937} t+\frac{0.01}{0.11} \sin \left(2 \pi \times \frac{0.11}{1000} t\right)\right]
\end{gathered}
$$

\subsection{Pulse rate variability analysis of the series and its evaluation}

PPIs were determined by simple positive peak detection assured by OriginPro; we did not use more complex detection algorithms like Peng and his colleagues [16] or Elgendi [29] considering the deterministic sinusoid-modulated cosine waveform in our investigation. Derivation that is a basic step in these more complex detection algorithms could result in another sinusoid wave just with a phase shift. The exported "tachograms" were the input of Varian 2.3 developed by the last author (LH) to perform HRV analysis from the master PPG signal, the decimated and the interpolated series. Table 1 displays the time and frequency domain parameters of the four master PPG signals.

Relative accuracy error (RAE) of the mean PPI (MPPI), its standard deviation (SDNN), and root mean square of successive PPI-differences (RMSSD) were calculated in the time domain; high (HF: 0.15-0.40 Hz) - and low (LF: 0.01$0.15 \mathrm{~Hz}$ ) - frequency integral in normalized units (NU) and $\mathrm{LF} / \mathrm{HF}$ ratio were computed by fast Fourier transformation (FFT) in the frequency domain. 


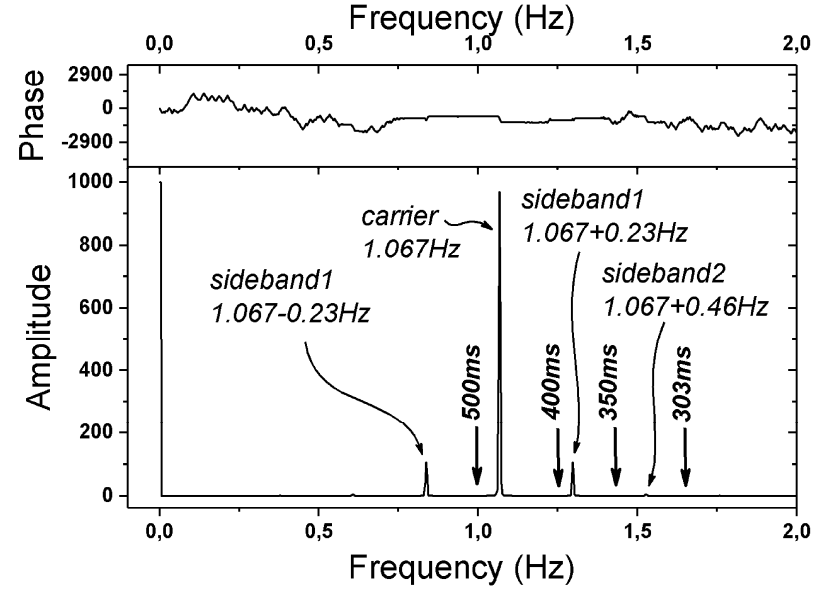

Fig.1. The spectral analysis of the sinusoidal frequency modulated signal (carrier: $1.067 \mathrm{~Hz}$, modulation at $0.23 \mathrm{~Hz}$, span $0.05 \mathrm{~Hz}$ ) illuminating the selection of certain higher values of decimation: cutting just below the carrier frequency $(500 \mathrm{~ms}$ decimation corresponding to $2 \mathrm{~Hz}$ sampling and $<1 \mathrm{~Hz}$ bandwidth), first side band (400 ms: $<1.25 \mathrm{~Hz}$ bandwidth), second side band $(350 \mathrm{~ms}$ : $<1.429 \mathrm{~Hz}$ ), and just above the second side band (303 ms: $<1.650 \mathrm{~Hz}$ ) considering the Nyquist-Shannon theorem with $>2 \mathrm{x}$ sampling frequency of the highest frequency component.

Also Poincaré-plots were created for visual comparison in the non-linear field, since it is a simple yet spectacular method, which sensitively detects outliers like, e.g., nonsinus beats in the ECG. In the entire study RAE $<5 \%$ was considered acceptable as common in biomedical sciences.

Table 1. Time- and frequency domain parameters of the four master PPG signals.

\begin{tabular}{|c|c|c|c|c|}
\hline $\begin{array}{l}\text { fmod / } \\
\text { span }\end{array}$ & $\begin{array}{l}0.23 \mathrm{~Hz} / \\
0.05 \mathrm{~Hz}\end{array}$ & $\begin{array}{l}0.23 \mathrm{~Hz} / \\
0.01 \mathrm{~Hz}\end{array}$ & $\begin{array}{l}0.11 \mathrm{~Hz} / \\
0.05 \mathrm{~Hz}\end{array}$ & $\begin{array}{|ll|}0.11 \mathrm{~Hz} / \\
0.01 \mathrm{~Hz} \\
\end{array}$ \\
\hline $\mathrm{n}$ & 319 & 319 & 319 & 319 \\
\hline $\operatorname{Max}(\mathrm{ms})$ & 980 & 946 & 982 & 946 \\
\hline $\operatorname{Min}(\mathrm{ms})$ & 897 & 929 & 895 & 928 \\
\hline MPPI (ms) & 937.138 & 937.028 & 937.151 & 937.031 \\
\hline SDNN (ms) & 28.784 & 5.805 & 30.513 & 6.115 \\
\hline RMSSD (ms) & 36.126 & 7.295 & 19.531 & 3.958 \\
\hline LF (NU) & 0.027 & 0.056 & 99.461 & 98.725 \\
\hline $\mathrm{HF}(\mathrm{NU})$ & 99.973 & 99.944 & 0.539 & 1.275 \\
\hline $\mathrm{LF} / \mathrm{HF}$ & 0.000 & 0.001 & 184.376 & 77.427 \\
\hline
\end{tabular}

Legend: The number (n) of detected peak-to-peak intervals (PPI), maximum (Max) and minimum (Min) PPI, mean PPI (MPPI), the standard deviation of PPIs (SDNN), the root mean square of the successive PPI differences (RMSSD) all in ms, HFNU - high frequency integral $(0.15-0.4 \mathrm{~Hz})$ in normalized units, LFNU - low frequency integral $(0.01-0.15 \mathrm{~Hz})$ in normalized units, $\mathrm{LF} / \mathrm{HF}-$ the ratio of $\mathrm{LF}$ and $\mathrm{HF}$.

\section{RESULTS}

\subsection{Time domain analysis of the decimated and the interpolated signals}

The results of the time domain analysis of the $0.23 \mathrm{~Hz}$ (respiratory arrhythmia) modulated signals at different sampling rates and consequent interpolation can be seen in Table 2. The peak-detection found all the 319 PPIs down to $200 \mathrm{~ms}$ sampling rate $(5 \mathrm{~Hz})$ after decimation, and $400 \mathrm{~ms}$ sampling rate $(2.50 \mathrm{~Hz})$ after interpolation in the "normal variability" series. The MPPI were within $5 \%$ RAE down to $303 \mathrm{~ms}(3.30 \mathrm{~Hz})$ in the decimated runs, whereas down to $400 \mathrm{~ms}(2.50 \mathrm{~Hz})$ sampling when interpolated back to $1 \mathrm{~ms}$ resolution. The SDNN required at least $20 \mathrm{~ms}(50 \mathrm{~Hz})$ sampling to remain within $5 \%$ error with no interpolation, which was improved down to $303 \mathrm{~ms}$ after interpolation. The RMSSD was more sensitive to the low sampling rate: without interpolation, $100 \mathrm{~Hz}$ was necessary to maintain the accuracy error below $5 \%$, whereas with interpolation $3.30 \mathrm{~Hz}$ was sufficient for that.

In the low variability series even at $303 \mathrm{~ms}$ all the peaks were found with no interpolation, however, interpolation improved peak detection $(\mathrm{n}=319)$ down to $400 \mathrm{~ms}$. The MPPI is extremely resistant even without interpolation repeating the sampling interval values seen at the normal variability series. The SDNN showed worse reproducibility at the reduced variability signals compared to the normal ones: at least $200 \mathrm{~Hz}$ sampling frequency was necessary with no interpolation versus the $5 \mathrm{~Hz}$, while it was $10 \mathrm{~Hz}$ versus $3.3 \mathrm{~Hz}$ with interpolation. The beat-to-beat variability parameter RMSSD was even more sensitive to the decimation: without interpolation $500 \mathrm{~Hz}$ was required for $<5 \%$ error. Interpolation improved it to $10 \mathrm{~Hz}$; however, at $2 \mathrm{~ms}$ and $10 \mathrm{~ms}$ sampling the RAE was above $6.5 \%$ probably due to certain rounding error or synchronization during the interpolation process resulting in an additional artificial variability (see later in 3.3. and in the discussion).

In the $0.11 \mathrm{~Hz}$ modulated series (Table 3.) with normal $(0.05 \mathrm{~Hz})$ span similar results were given like at $0.23 \mathrm{~Hz}$ modulation series, however, the RMSSD with or without interpolation showed greater sensitivity to the sampling as a consequence of the relatively lower beat-to-beat variability because of the lower modulation frequency at $0.11 \mathrm{~Hz}$. In the low-span $(0.01 \mathrm{~Hz})$ group at $0.11 \mathrm{~Hz}$ the SDNN needs similar sampling interval to the one at normal variability series, whilst the RMSSD could not be reproduced within $<5 \%$ accuracy even at $500 \mathrm{~Hz}$ sampling frequency due to the very small beat-to-beat variability. Interpolation at 20,50, and $100 \mathrm{~ms}$ restored the accuracy of RMSSD, interestingly it failed at 2, 5, and $10 \mathrm{~ms}$ decimation (see later in 3.3. and in the discussion).

\subsection{Frequency domain analysis of the decimated and the interpolated signals}

The $0.23 \mathrm{~Hz}$ modulated signal contains exclusively $\mathrm{HF}$ band after removing the DC component (the mean) on spectral analysis by Varian, whereas the $0.11 \mathrm{~Hz}$ modulated series includes just the LF band. 
Table 2. Relative accuracy error in the time domain analysis of decimated (D) and interpolated (I) series with $0.05 \mathrm{~Hz}$ and $0.01 \mathrm{~Hz}$ deviation at $0.23 \mathrm{~Hz}$ modulation in regards to the sampling interval (SI).

\begin{tabular}{l|ll|ll|ll|ll}
\multicolumn{8}{c}{ PPI=937 ms, fmod=0.23 Hz, span=0.05 Hz } \\
$\mathbf{S I}(\mathbf{m s})$ & $\mathbf{n}(\mathbf{D})$ & $\mathbf{n}(\mathbf{I})$ & MPPI (D) & MPPI (I) & SDNN(D) & SDNN(I) & RMSSD(D) & RMSSD(I) \\
\hline $\mathbf{1}$ & $\underline{\mathbf{3 1 9}}$ & & $\underline{\mathbf{9 3 7 . 1 3 8}}$ & & $\underline{\mathbf{2 8 . 7 8 4}}$ & & $\underline{\mathbf{3 6 . 1 2 6}}$ & \\
$\mathbf{2}$ & 319 & 319 & 0.000 & 0.000 & 0.216 & 0.905 & 0.240 & 1.068 \\
$\mathbf{5}$ & 319 & 319 & -0.001 & 0.000 & 0.394 & -0.068 & 0.617 & 0.027 \\
\hline $\mathbf{1 0}$ & 319 & 319 & -0.002 & 0.000 & 1.782 & -0.113 & 2.674 & -0.040 \\
$\mathbf{2 0}$ & 319 & 319 & 0.004 & 0.000 & 2.547 & -0.278 & $\mathbf{5 . 0 9 7}$ & -0.261 \\
$\mathbf{5 0}$ & 319 & 319 & 0.001 & 0.000 & $\mathbf{2 2 . 7 9 9}$ & -0.141 & $\mathbf{3 8 . 1 8 8}$ & -0.154 \\
\hline $\mathbf{1 0 0}$ & 319 & 319 & 0.018 & 0.000 & $\mathbf{6 8 . 2 7 8}$ & -0.115 & $\mathbf{1 1 1 . 1 3 3}$ & -0.117 \\
$\mathbf{2 0 0}$ & 319 & 319 & 0.018 & 0.001 & $\mathbf{2 2 2 . 8 4 2}$ & 0.505 & $\mathbf{3 3 9 . 0 5 1}$ & 1.677 \\
$\mathbf{3 0 3}$ & $\mathbf{3 0 9}$ & 319 & 2.857 & -0.004 & $\mathbf{4 7 4 . 8 5 3}$ & 1.324 & $\mathbf{4 7 7 . 4 6 7}$ & 0.321 \\
\hline $\mathbf{3 5 0}$ & $\mathbf{2 1 5}$ & 319 & $\mathbf{4 7 . 4 8 0}$ & 0.025 & $\mathbf{1 1 1 7 . 1 9 0}$ & $\mathbf{7 6 . 7 3 0}$ & $\mathbf{1 6 6 7 . 1 9 7}$ & $\mathbf{1 2 8 . 0 3 9}$ \\
$\mathbf{4 0 0}$ & $\mathbf{1 0 8}$ & 319 & $\mathbf{1 9 2 . 4 5 9}$ & 0.064 & $\mathbf{1 3 0 6 . 6 2 9}$ & $\mathbf{1 9 9 . 5 1 3}$ & $\mathbf{1 8 9 6 . 8 3 2}$ & $\mathbf{3 0 8 . 9 5 8}$ \\
$\mathbf{5 0 0}$ & $\mathbf{3 9}$ & $\mathbf{2 7 8}$ & $\mathbf{6 9 2 . 1 0 1}$ & $\mathbf{1 4 . 6 5 2}$ & $\mathbf{1 5 6 8 . 9 2 3}$ & $\mathbf{2 4 2 . 9 8 0}$ & $\mathbf{2 0 5 3 . 5 5 4}$ & $\mathbf{2 5 4 . 4 7 8}$
\end{tabular}

\begin{tabular}{l|cc|cc|cc|c|c}
\multicolumn{8}{c}{ PPI=937 ms, fmod=0.23 Hz, span=0.01 Hz } \\
SI (ms) & $\mathbf{n}(\mathbf{D})$ & $\mathbf{n}(\mathbf{I})$ & MPPI (D) & MPPI (I) & SDNN(D) & SDNN(I) & RMSSD(D) & RMSSD(I) \\
\hline$\underline{\mathbf{1}}$ & $\underline{\mathbf{3 1 9}}$ & & $\underline{\mathbf{9 3 7 . 0 2 8}}$ & & $\underline{\mathbf{5 . 8 0 5}}$ & & $\underline{\mathbf{7 . 2 9 5}}$ & \\
$\mathbf{2}$ & 319 & 319 & 0.000 & 0.000 & 0.753 & 4.482 & 1.560 & $\mathbf{6 . 5 9 1}$ \\
$\mathbf{5}$ & 319 & 319 & -0.001 & -0.001 & 3.459 & 1.492 & $\mathbf{6 . 7 8 9}$ & 2.702 \\
\hline $\mathbf{1 0}$ & 319 & 319 & -0.001 & -0.001 & $\mathbf{2 0 . 1 5 1}$ & 3.729 & $\mathbf{3 4 . 2 4 6}$ & $\mathbf{6 . 5 4 5}$ \\
$\mathbf{2 0}$ & 319 & 319 & 0.003 & 0.000 & $\mathbf{7 2 . 8 8 1}$ & -0.060 & $\mathbf{1 1 9 . 5 8 1}$ & 0.710 \\
$\mathbf{5 0}$ & 319 & 319 & 0.013 & 0.000 & $\mathbf{2 7 6 . 9 8 6}$ & -0.589 & 392.199 & -0.439 \\
\hline $\mathbf{1 0 0}$ & 319 & 319 & -0.004 & 0.000 & $\mathbf{7 3 2 . 9 3 8}$ & -1.051 & $\mathbf{1 0 7 8 . 3 7 3}$ & -0.762 \\
$\mathbf{2 0 0}$ & 319 & 319 & 0.029 & 0.002 & $\mathbf{1 5 0 0 . 7 6 0}$ & $\mathbf{2 4 . 8 7 1}$ & $\mathbf{2 0 7 4 . 1 7 3}$ & $\mathbf{4 2 . 3 6 2}$ \\
$\mathbf{3 0 3}$ & 319 & 319 & -0.052 & -0.005 & $\mathbf{1 4 0 2 . 8 4 6}$ & $\mathbf{6 3 . 0 2 3}$ & $\mathbf{1 6 7 3 . 8 0 5}$ & $\mathbf{1 6 . 5 4 1}$ \\
\hline $\mathbf{3 5 0}$ & $\mathbf{2 1 5}$ & 319 & $\mathbf{4 7 . 4 9 7}$ & 0.033 & $\mathbf{5 9 3 5 . 2 3 2}$ & $\mathbf{6 3 7 . 7 9 7}$ & $\mathbf{9 2 6 8 . 4 7 8}$ & $\mathbf{8 8 8 . 5 0 1}$ \\
$\mathbf{4 0 0}$ & $\mathbf{1 0 8}$ & 319 & $\mathbf{1 9 2 . 4 9 3}$ & 0.199 & $\mathbf{3 5 2 5 . 8 8 5}$ & $\mathbf{1 3 5 3 . 4 5 7}$ & $\mathbf{4 1 4 0 . 5 3 8}$ & $\mathbf{1 9 1 8 . 0 7 1}$ \\
$\mathbf{5 0 0}$ & $\mathbf{3 9}$ & $\mathbf{2 7 8}$ & $\mathbf{6 9 4 . 9 3 0}$ & $\mathbf{1 4 . 6 7 0}$ & $\mathbf{3 7 4 9 . 2 3 7}$ & $\mathbf{1 5 2 4 . 9 5 0}$ & $\mathbf{4 3 4 7 . 3 4 6}$ & $\mathbf{1 5 6 2 . 9 7 7}$
\end{tabular}

Legend: At the $1 \mathrm{~ms}$ SI the master PPGs' parameters are given in ms. The number (n) of detected peak-to-peak intervals (PPI), the mean PPI (MPPI), the standard deviation of PPIs (SDNN), the root mean square of the successive PPI differences (RMSSD). Totally $n=319+1$ peaks were generated resulting in $320 * 937 \mathrm{~ms}=299.840 \mathrm{~s}$ record length as required in standard short-term heart rate variability analysis. Highlighted with bald italics when RAE $>5 \%$.

Accordingly, at the $0.23 \mathrm{~Hz}$ modulated series the LF component is negligible, that is why decimating even at $2 \mathrm{~ms}$ results in extremely high accuracy error $(>7.407$, that is over $700 \%$ ), which cannot be reconstructed by cubic spline interpolation. Interestingly, by interpolation of $20 \mathrm{~ms}$ and $50 \mathrm{~ms}$ decimated series, the RAE improved to $0.000 \%$ at $0.05 \mathrm{~Hz}$ span, and also in the $0.01 \mathrm{~Hz}$ span series at these sampling intervals the RAE was smaller. The reason for this artefact must be rounding error, probably due to the $20 \mathrm{~Hz} *$ $50 \mathrm{~Hz}=1000 \mathrm{~Hz}$ original sampling rate but not the synchronization of the $937 \mathrm{~ms}$ PPI. LF results are repeated also in LF/HF ratio. The entire variability of this signal is in the HF range, therefore this band in this series is more resistant to low sampling, resulting in $\mathrm{RAE}<5 \%$ even at $100 \mathrm{~ms}$ sampling without interpolation and down to $400 \mathrm{~ms}$ with interpolation. When the modulation frequency is $0.11 \mathrm{~Hz}$ falling into the LF band of variability, LF and HF parameters flip in magnitude compared to the $0.23 \mathrm{~Hz}$ modulation series, including the negligible accuracy error of LF at 20-50-100 ms. The LFNU is correct within $5 \%$ RAE with no interpolation down to $20 \mathrm{~ms}$, while with interpolation it increases up to $200 \mathrm{~ms}$ at normal variability, however, with reduced variability these intervals are $5 \mathrm{~ms}$ and $100 \mathrm{~ms}$, respectively. The HF band is more sensitive to the low sampling rates than the LF band in the relevant modulation series. 
Table 3. Relative accuracy error in the time domain analysis of decimated (D) and interpolated (I) series with $0.05 \mathrm{~Hz}$ and $0.01 \mathrm{~Hz}$ deviation at $0.11 \mathrm{~Hz}$ modulation in regards to the sampling interval (SI).

\begin{tabular}{l|cc|cc|cc|cc}
\multicolumn{7}{c}{ PPI=937 ms, fmod=0.11 Hz, span=0.05 Hz } \\
SI (ms) & $\mathbf{n}(\mathbf{D})$ & $\mathbf{n}(\mathbf{I})$ & MPPI (D) & MPPI (I) & SDNN(D) & SDNN(I) & RMSSD(D) & RMSSD(I) \\
\hline $\mathbf{1}$ & $\underline{\mathbf{3 1 9}}$ & & $\underline{\mathbf{9 3 7 . 1 5 1}}$ & & $\underline{\mathbf{3 0 . 5 1 3}}$ & & $\underline{\mathbf{1 9 . 5 3 1}}$ & \\
$\mathbf{2}$ & 319 & 319 & 0.000 & 0.000 & 0.057 & 0.042 & 0.203 & 0.722 \\
$\mathbf{5}$ & 319 & 319 & 0.000 & -0.001 & 0.182 & 0.019 & 1.787 & 0.744 \\
\hline $\mathbf{1 0}$ & 319 & 319 & 0.000 & 0.000 & 1.280 & -0.031 & $\mathbf{6 . 3 9 2}$ & 0.881 \\
$\mathbf{2 0}$ & 319 & 319 & 0.003 & 0.000 & 2.828 & 0.048 & $\mathbf{2 0 . 4 5 6}$ & 0.184 \\
$\mathbf{5 0}$ & 319 & 319 & 0.000 & 0.000 & $\mathbf{2 0 . 1 3 4}$ & 0.050 & $\mathbf{1 0 6 . 0 5 1}$ & 0.095 \\
\hline $\mathbf{1 0 0}$ & 319 & 319 & 0.016 & 0.000 & $\mathbf{6 0 . 8 5 6}$ & 0.069 & $\mathbf{2 5 6 . 3 1 3}$ & 0.112 \\
$\mathbf{2 0 0}$ & 319 & 319 & 0.016 & 0.002 & $\mathbf{2 0 4 . 5 4 8}$ & 0.831 & $\mathbf{7 0 8 . 0 4 1}$ & $\mathbf{8 . 3 8 4}$ \\
$\mathbf{3 0 3}$ & $\mathbf{3 1 5}$ & 319 & 0.999 & -0.004 & $\mathbf{2 8 8 . 9 4 2}$ & 3.920 & $\mathbf{7 9 7 . 1 3 3}$ & $\mathbf{1 4 . 5 7 6}$ \\
\hline $\mathbf{3 5 0}$ & $\mathbf{2 1 5}$ & 319 & $\mathbf{4 7 . 4 7 8}$ & 0.027 & $\mathbf{1 0 4 8 . 2 1 6}$ & $\mathbf{7 3 . 3 9 9}$ & $\mathbf{2 9 1 0 . 6 7 6}$ & $\mathbf{3 0 8 . 1 6 9}$ \\
$\mathbf{4 0 0}$ & $\mathbf{1 0 8}$ & 319 & $\mathbf{1 9 0 . 8 7 4}$ & 0.198 & $\mathbf{1 7 4 6 . 5 0 9}$ & $\mathbf{1 8 5 . 8 4 7}$ & $\mathbf{4 7 1 7 . 4 0 8}$ & $\mathbf{6 3 8 . 9 5 2}$ \\
$\mathbf{5 0 0}$ & $\mathbf{3 9}$ & $\mathbf{2 7 8}$ & $\mathbf{6 9 4 . 8 2 6}$ & $\mathbf{1 4 . 6 4 6}$ & $\mathbf{3 0 8 5 . 3 1 3}$ & $\mathbf{2 2 1 . 2 4 0}$ & $\mathbf{7 1 8 8 . 4 9 7}$ & $\mathbf{5 2 7 . 2 0 4}$
\end{tabular}

\begin{tabular}{l|cc|cc|cc|cc}
\multicolumn{8}{c}{ PPI=937 ms, fmod=0.11 Hz, span=0.01 Hz } \\
SI (ms) & $\mathbf{n}(\mathbf{D})$ & $\mathbf{n}(\mathbf{I})$ & MPPI (D) & MPPI (I) & SDNN(D) & SDNN(I) & RMSSD(D) & RMSSD(I) \\
\hline $\mathbf{1}$ & $\underline{\mathbf{3 1 9}}$ & & $\underline{\mathbf{9 3 7 . 0 3 1}}$ & & $\underline{\mathbf{6 . 1 1 5}}$ & & $\underline{\mathbf{3 . 9 5 8}}$ & \\
$\mathbf{2}$ & 319 & 319 & 0.000 & 0.000 & 0.792 & 2.108 & $\mathbf{5 . 8 0 4}$ & $\mathbf{1 3 . 0 7 8}$ \\
$\mathbf{5}$ & 319 & 319 & -0.001 & 0.000 & 4.648 & 0.999 & $\mathbf{3 0 . 6 3 4}$ & $\mathbf{8 . 7 7 0}$ \\
\hline $\mathbf{1 0}$ & 319 & 319 & -0.001 & 0.000 & $\mathbf{1 8 . 4 1 0}$ & 2.425 & $\mathbf{9 9 . 3 7 8}$ & $\mathbf{2 0 . 1 2 0}$ \\
$\mathbf{2 0}$ & 319 & 319 & 0.002 & 0.000 & $\mathbf{6 6 . 1 6 8}$ & 0.661 & $\mathbf{2 7 8 . 0 8 1}$ & 4.275 \\
$\mathbf{5 0}$ & 319 & 319 & 0.012 & 0.000 & $\mathbf{2 5 7 . 9 0 5}$ & 0.301 & $\mathbf{8 0 7 . 2 6 7}$ & 1.008 \\
\hline $\mathbf{1 0 0}$ & 319 & 319 & -0.004 & 0.000 & $\mathbf{6 9 0 . 7 7 8}$ & 0.486 & $\mathbf{2 0 7 2 . 0 8 7}$ & 2.302 \\
$\mathbf{2 0 0}$ & 319 & 319 & 0.029 & 0.002 & $\mathbf{1 4 1 9 . 7 3 6}$ & $\mathbf{2 4 . 5 2 6}$ & $\mathbf{3 9 0 7 . 6 3 8}$ & $\mathbf{1 1 8 . 3 8 4}$ \\
$\mathbf{3 0 3}$ & 319 & 319 & -0.052 & -0.004 & $\mathbf{1 3 2 6 . 7 7 8}$ & $\mathbf{5 8 . 3 8 6}$ & $\mathbf{3 1 6 9 . 6 4 1}$ & $\mathbf{5 8 . 1 3 0}$ \\
\hline $\mathbf{3 5 0}$ & $\mathbf{2 1 5}$ & 319 & $\mathbf{4 7 . 4 9 7}$ & 0.017 & $\mathbf{5 6 2 9 . 7 5 5}$ & $\mathbf{6 0 0 . 8 8}$ & $\mathbf{1 6 9 5 5 . 9 1}$ & $\mathbf{1 7 1 6 . 0 1}$ \\
$\mathbf{4 0 0}$ & $\mathbf{1 0 8}$ & 319 & $\mathbf{1 9 1 . 7 0 1}$ & $\mathbf{8 5 . 5 8 8}$ & $\mathbf{4 3 2 6 . 8 1 1}$ & $\mathbf{1 2 1 4 0 . 4 9}$ & $\mathbf{1 1 1 2 5 . 6 7}$ & $\mathbf{2 5 5 4 9 . 0 8}$ \\
$\mathbf{5 0 0}$ & $\mathbf{3 9}$ & $\mathbf{2 7 8}$ & $\mathbf{6 9 2 . 1 9 1}$ & $\mathbf{1 4 . 6 6 9}$ & $\mathbf{5 6 9 3 . 7 1 7}$ & $\mathbf{1 4 4 0 . 3 4}$ & $\mathbf{1 2 8 6 1 . 8 0}$ & $\mathbf{2 9 5 0 . 6 9}$
\end{tabular}

Legend: At the $1 \mathrm{~ms}$ SI the master PPGs' parameters are given in ms. The number (n) of detected peak-to-peak intervals (PPI), the mean PPI (MPPI), the standard deviation of PPIs (SDNN), the root mean square of the successive PPI differences (RMSSD). Highlighted with bald italics when RAE $>5 \%$.

3.3. Poincaré-plot visual analysis of the decimated and the interpolated signals

Fig.2. shows the Poincaré-plots of the tachograms of the original, decimated and interpolated PPG signals. In the first and third column the effects of decimation clearly show the dispersion and the coarse-grained structure of the cloud inversely proportional to the temporal resolution of the given PPG signal. Without interpolation $2 \mathrm{~ms}$ decimation is visually acceptable regarding the shape of the cloud. The scale of the plot (990 ms) changed from the $50 \mathrm{~ms}$-decimated plots due to the $1000 \mathrm{~ms}$ maxima. Above $303 \mathrm{~ms}$ resolution $(3.3 \mathrm{~Hz})$ there is a jump in the scale because of undetected peaks and consequent two or more times longer PPIs.
SDNN and RMSSD both can be restored within $5 \%$ error down to $303 \mathrm{~ms}$ in the $0.23 \mathrm{~Hz}$ modulated normal variability series; however, the fine structure of the Poincaré-cloud could be reconstructed just down to $100 \mathrm{~ms}$. At 200-500 ms decimation and consecutive $1 \mathrm{~ms}$ interpolation the shape of the Poincaré-plot is significantly different in spite of good reproduction of time domain parameters at $200 \mathrm{~ms}$ and $303 \mathrm{~ms}$. Additionally, at 2-5-10 ms decimation and interpolation the dispersion of the Poincaré-cloud (here ring) is higher compared to the original or the 20-50-100 ms ones, which is reflected in the RAE of beat-to-beat RMSSD as well. This reflects less precise interpolation when fewer than 10 points are inserted. 

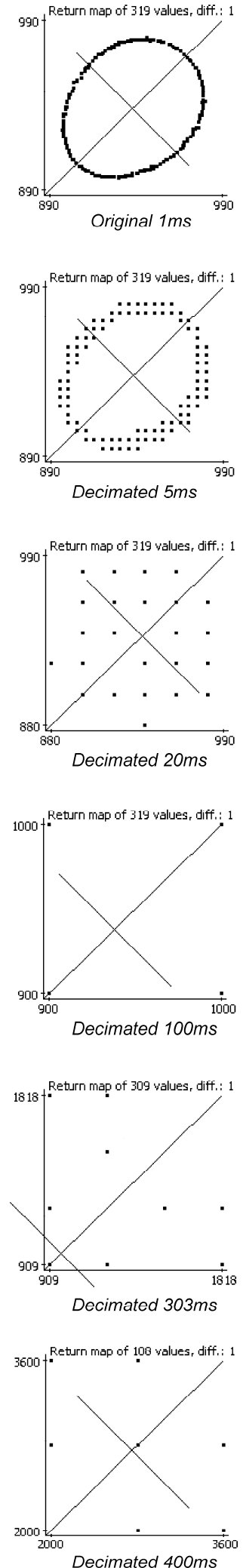
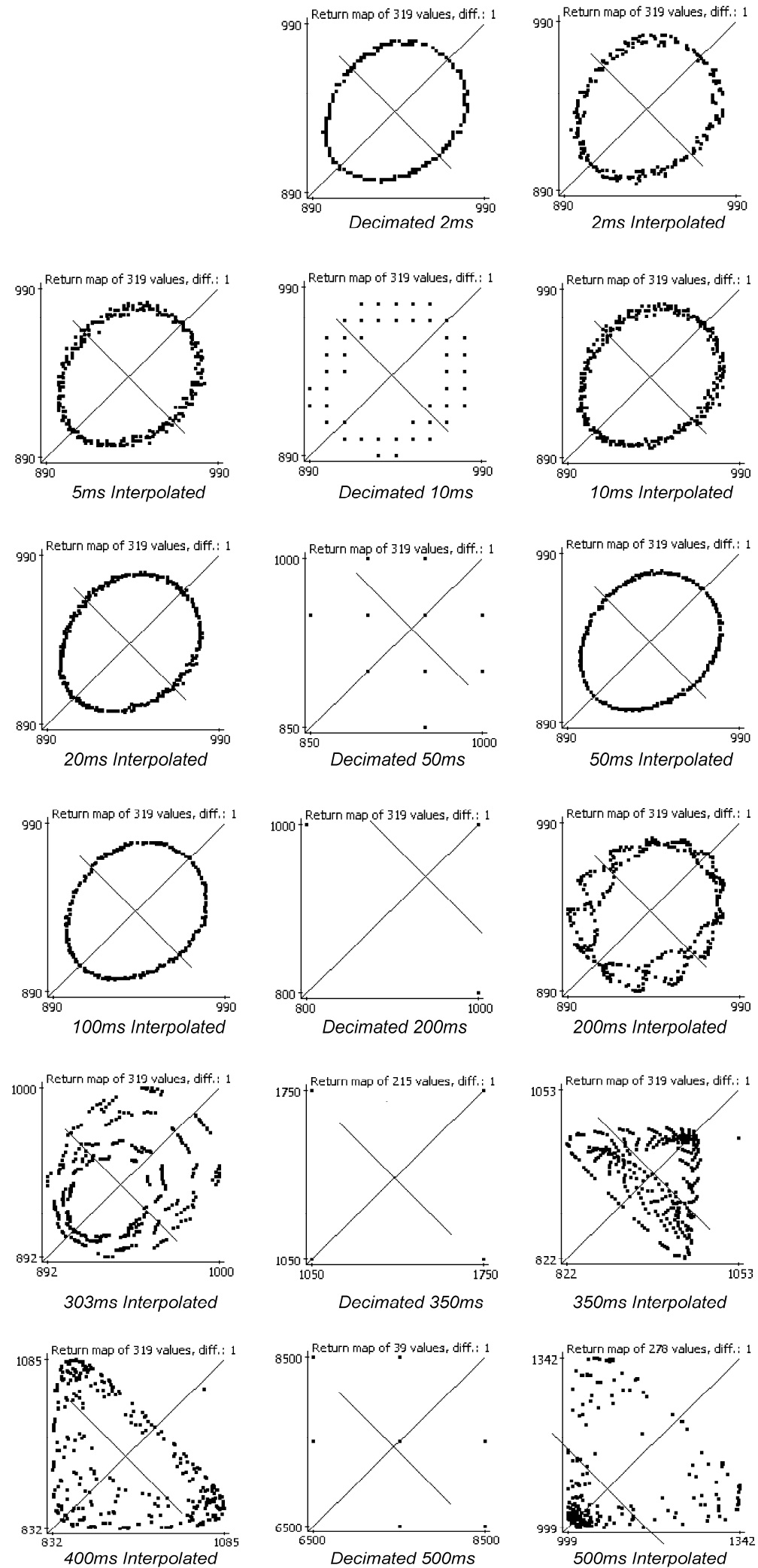

Fig.2. Poincaré-plots at different decimation values and subsequent cubic spline interpolation compared to the original one (left upper corner with $1 \mathrm{~ms}$ resolution, carrier: $1.067 \mathrm{~Hz}$, modulation at $0.23 \mathrm{~Hz}$, span $0.05 \mathrm{~Hz}$ ). The coarser texture of the cloud is clearly visible as the decimation interval increases (in the first and third columns from the left). Interpolation restores the "ring" up to $100 \mathrm{~ms}$ decimation; however, time domain parameters are reproduced within $5 \%$ relative accuracy error up to $303 \mathrm{~ms}$. In spite of this, at $200 \mathrm{~ms}$ and $303 \mathrm{~ms}$ the regular ring structure cannot be reconstructed by interpolation. Another interesting observation is that at 2-5-10 ms interpolation the ring is more dispersed compared to the original and the 20-50-100 ms interpolation (The plots are directly saved from Varian 2.3 with its own fixed resolution). 


\section{DISCUSSION}

Above results demonstrate apparent evidence that the reliability of HRV parameters depends on the original variability of the signal, the sampling interval, the possible interpolation, and the investigated parameter as well. Inherently reduced variability needs a higher sampling rate since sampling error proportional to sampling interval is additive to natural variability. Interpolation can significantly improve the accuracy of HRV measures. Generally, time domain parameters are more resistant to low sampling frequency: mean $>$ SDNN $>$ RMSSD, in decreasing order. RMSSD is the most susceptible to the sampling error due to its beat-to-beat-weighted sensitivity, while the MPPI eliminates the sampling error (uniformly distributed random noise with a zero mean) by averaging. The frequency domain analysis could be interpreted with limitations since the $0.23 \mathrm{~Hz}$ modulated series had no LF component while the other series had no HF component, which results in extremely high accuracy error in the missing band due to the ratio of its power approximating zero and the non-zero sampling error. These results can be clearly explained by the spectral analysis of the modulated signal (Fig.1.): cutting below the carrier frequency (equivalent to $937 \mathrm{~ms}$ ) even the MPPI cannot be reconstructed by interpolation. Cutting between the carrier and the first right side band defined by the $400 \mathrm{~ms}$ decimation, the decimated mean is incorrect, however, after interpolation it is well below $1 \%$ error (except at $0.11 \mathrm{~Hz}$ with $0.01 \mathrm{~Hz}$ deviation). In normal variability series keeping two sidebands $(1.067 \mathrm{~Hz}+0.23 \mathrm{~Hz}+0.23 \mathrm{~Hz}=1.527 \mathrm{~Hz}$ corresponding to $303 \mathrm{~ms}$ ) can restore SDNN and RMSSD values within $5 \%$ RAE. In the low variability series more sidebands are required to be preserved resulting in the $100 \mathrm{~ms}$ minimal sampling with consecutive interpolation.

In the low-variability series at $2-5-10 \mathrm{~ms}$ decimation and interpolation the RAE of RMSSD is interestingly higher compared to the original or the $20-50-100 \mathrm{~ms}$ runs. It is also reflected in the dispersion of the Poincaré-"ring" at the given decimation and interpolation. It means there is an artefact of cubic spline interpolation in the nonlinear structure but not in time domain parameters, if less than 10 points are inserted. It calls attention to the potential failing of cubic spline interpolation.

According to Baek et al. [25] PPG signal can be sampled at $20 \mathrm{~Hz}$ in order to "assess trends" in time- and frequency domain as well as in non-linear parameters when the temporal resolution is enhanced by parabola approximation or cubic spline interpolation; nonetheless it should be mentioned that the "absolute HRV values were not exactly matched to the reference signal's values" in the research. By Mahdiani et al. [26] down-sampling the ECG even to $50 \mathrm{~Hz}$ with subsequent cubic interpolation results in almost accurate time domain parameters compared to the original $5 \mathrm{kHz}$-sampled signals.

Choi and Shin [27] allow extremely low sampling frequency of $25 \mathrm{~Hz}$ for PPG signal acquisition without interpolation and with a single-point peak detection algorithm based on their pooled statistical analysis. At the level of the individual their results can be questionable considering the \pm 20 ms uniformly distributed random noise as sampling error belonging to $25 \mathrm{~Hz}$ sampling rate, which is added to each fiducial point (the peaks in this study), those defining the pulse rate interval. They pled their results by the sampling theorem and the dominantly $<10 \mathrm{~Hz}$ frequency content of the power spectrum of the PPG signal.

We would like to call attention to the discordance of the minimally necessary sampling frequency without interpolation demonstrated by our results and the theoretical Nyquist-frequency defined by the concrete signal bandwidth. The Shannon or Nyquist-Shannon sampling theorem is often referenced incorrectly (e.g., in [27]) regarding the useful bandwidth of biological signals and the minimally required sampling frequency that should be greater than two times the highest frequency component in the analogue signal in order to correctly reconstruct the original signal. This latter part in italics is often ignored: reproduction by a low-pass filter converts back the discrete series into continuous signal, namely "fills the spaces" or "interpolates" between the impulses. It is not stated in any adaptation of the theorem that the original and the sampled signals are equivalent. Claude $\mathrm{E}$. Shannon, the father of information theory published his theoretical paper in 1948, before the era of digital computers and analogue-to-digital conversion [30]. He investigated the problem of best encoding of the information for transmission by chopping the signal to short segments [31]. Shannon discussed also the reconstruction with sync-functions referring to the Whittaker-Shannon interpolation formula from 1915. Today we are able to display and work directly with discrete signals in the digital domain; however, we disregard the sampling error or uncertainty of the discrete points representing the real-world continuous signal. The reconstruction filter in the hardware from Shannon's era should be replaced today by interpolation methods on the software side. However, it will never be a real continuous signal inside a digital computer, just a series of impulse trains with sufficient temporal resolution optimized to the given task. The appropriately selected sampling frequency (usually well above the expected Nyquist frequency) can assure sufficient temporal resolution for further direct processing without interpolation.

Based on the power spectrum of our modulated sinusoid wave (Fig.1.), one can estimate the minimally necessary signal bandwidth and sampling frequency specifically for the given proband population: e.g., for a professional sportsman with $\sim 200$ beat $/ \mathrm{min}$ pulse rate and $\sim 40 / \mathrm{min}$ breathing rate the PPG bandwidth must be at least $1 / 0.3 \mathrm{~s}+3 * 1 / 1.5 \mathrm{~s}=5.33 \mathrm{~Hz}$ with preserving three sidebands, and consequently $>10.66 \mathrm{~Hz}$ sampling frequency is needed with interpolation.

The Poincaré-plot can be a useful tool for assessing the accurateness and precision of different interpolation or other signal processing methods. It is more sensitive to artefacts than time domain (standard statistical) parameters according to our observations.

Study limitations: Some study limitations should be mentioned as well: we investigated a deterministic periodic signal with discrete spectrum versus the quasi-periodic, chaotic real PPG with continuous spectrum. The NyquistShannon sampling theorem does not specify any boundary condition of the given signal except its highest frequency component; therefore, our observations must apply to real 
PPGs as well. Another difference is the asymmetric acceleration and deceleration of the real pulse rate opposing the symmetrical ascension and fall of our artificial PPG series. Cubic spline interpolation works ideally on symmetric curves like in our study, so in real PPGs also, e.g., parabola approximation should be tested [26]. Another limitation may be the single fixed 5-minute length of the synthesized records according to short-term HRV analysis standards [28]; however, also shorter periods can be applied occasionally, especially for mean pulse rate calculation.

Novelties: 1: Present investigation confirmed that higher sampling rate is required for accurate HRV analysis than the Nyquist frequency. 2: Minimally necessary sampling frequency depends on the proband's intrinsic variability, the interpolation, and the given parameter. 3: Based on the power spectrum of the present model, the minimal bandwidth and sampling rate can be calculated from the expected heart rate, breathing rate and variability. 4: Poincaré plot can detect delicate imprecision of digital signal processing.

\section{CONCLUSION}

Current study defined the minimally necessary sampling frequency of computer simulated variable PPG signals with different variability and modulation frequencies in order to maintain accuracy within $5 \%$ RAE of the parameters in the time and frequency domain, and to preserve the Poincaré-plot structure. The MPPI is extremely resistant to the low sampling frequency down to $3.30 \mathrm{~Hz}$ without interpolation, and down to $2.50 \mathrm{~Hz}$ with cubic spline interpolation. The SDNN requires $50 \mathrm{~Hz}$ sampling with normal variability, while it needs at least $200 \mathrm{~Hz}$ in the low-variability samples without interpolation. Interpolation improves the RAE of SDNN underneath $5 \%$ down to $3.30 \mathrm{~Hz}$ in normal and $10 \mathrm{~Hz}$ in the reduced variability series. The RMSSD acts more sensitively: without interpolation $100 \mathrm{~Hz}$ in the normal, whereas $500 \mathrm{~Hz}$ in the reduced variability series is necessary. With interpolation, above numbers are $3.30 \mathrm{~Hz}$ and $10 \mathrm{~Hz}$, respectively, regarding $0.23 \mathrm{~Hz}$ modulated signals.

In the frequency domain analysis without interpolation $200 \mathrm{~Hz}$ sampling frequency is recommended in order to get accurate values even in low-variability series, while with cubic spline interpolation $10 \mathrm{~Hz}$ can be sufficient with present boundaries.

On the Poincaré-plot with no interpolation $2 \mathrm{~ms}$, with interpolation $100 \mathrm{~ms}$ sampling interval was required to preserve visually the shape of the plot, however, at $200 \mathrm{~ms}$ and $303 \mathrm{~ms}$ the morphology was totally different with interpolation in spite of accurate time domain parameters. This Poincaré-plot method can also be a sensitive tool for the assessment of accurateness of different interpolation or other signal processing techniques.

Above results obviously highlight that the necessary sampling frequency is well above the $2 \mathrm{x}$ bandwidth (Nyquist frequency), especially in the low-variability series, calling attention to the ratio of the real HRV and the sampling error proportional to the sampling interval.

When choosing the optimal sampling frequency in lowpower wearable or mobile instrumentation, the probands' expected mean pulse rate and heart rate variability, the sensitivity of the actually measured parameters, the application of some interpolation technique, and the pulse rate detection method (curve fitting or single point based) all should be taken into account. Based on the conclusions of present investigation, further studies are encouraged on real PPGs.

\section{ACKNOWLEDGMENT}

The work was supported by AOK-KA/2017 Grant from the Medical Faculty, University of Pécs, Hungary.

\section{REFERENCES}

[1] Bunn, J.A., Navalta, J.W, Fountaine, C.J., Reece, J.D. (2018). Current state of commercial wearable technology in physical activity monitoring 2015-2017. International Journal of Exercise Science, 11 (7), 503515.

[2] Liu, Y., Wang, H., Zhao, W., Zhang, M., Qin, H., Xie, Y. (2018). Flexible, stretchable sensors for wearable health monitoring: Sensing mechanisms, materials, fabrication strategies and features. Sensors (Basel), 18 (2), 645 .

[3] Kumar, A., Komaragiri, R., Kumar, M. (2018). From pacemaker to wearable: Techniques for ECG detection systems. Journal of Medical Systems, 42 (2), 34.

[4] Pinheiro, E., Postolache, O. (2008). A wireless monitoring system for health care applications. In The Sixth IASTED International Conference on Biomedical Engineering, 13-15 February 2008, Innsbruck, Austria. Acta Press, 372-377.

[5] Pollonini, L., Rajan, N.O., Xu, S., Madala, S., Dacso, C.C. (2010). A novel handheld device for use in remote patient monitoring of heart failure patients - design and preliminary validation on healthy subjects. Journal of Medical Systems, 36 (2), 653-659.

[6] Silva, I., Moody, G.B., Celi, L. (2011). Improving the quality of ECGs collected using mobile phones: The PhysioNet/Computing in Cardiology Challenge 2011. Computing in Cardiology, 38, 273-276.

[7] Quintana, D.S., Heathers, J.A., Kemp, A.H. (2012). On the validity of using the Polar RS800 heart rate monitor for heart rate variability research. European Journal of Applied Physiology, 112 (12), 4179-4180.

[8] Kovács, L., Kézér, F.L., Jurkovich, V., KulcsárHuszenicza, M., Tőzsér, J. (2015). Heart rate variability as an indicator of chronic stress caused by lameness in dairy cows. PLoS One, 10 (8), e0134792.

[9] Bouts, A.M., Brackman, L., Martin, E., Subasic, A.M., Potkanowicz, E.S. (2018). The accuracy and validity of iOS-based heart rate apps during moderate to high intensity exercise. International Journal of Exercise Science, 11 (7), 533-540.

[10] Shcherbina, A., Mattsson, C.M., Waggott, D., Salisbury, H., Christle, J.W., Hastie, T., Wheeler, M.T., Ashley, E.A. (2017). Accuracy in wrist-worn, sensorbased measurements of heart rate and energy expenditure in a diverse cohort. Journal of Personalized Medicine, 7 (2). 
[11] Stahl, S.E., An, H.S., Dinkel, D.M., Noble, J.M., Lee, J.M. (2016). How accurate are the wrist-based heart rate monitors during walking and running activities? Are they accurate enough? BMJ Open Sport and Exercise Medicine, 2 (1), e000106.

[12] Wallen, M.P., Gomersall, S.R., Keating, S.E., Wisløff, U., Coombes, J.S. (2016). Accuracy of heart rate watches: Implications for weight management. PLoS One, 11 (5), e0154420.

[13] Atlasz, T., Kellényi, L., Kovács, P., Babai, N., Thuróczy, G., Hejjel, L., Hernádi, I. (2006) The application of surface plethysmography for heart rate variability analysis after GSM radiofrequency exposure. Journal of Biochemical and Biophysical Methods, 69 (1-2), 233-236.

[14] Spierer, D.K., Rosen, Z., Litman, L.L., Fujii, K. (2015). Validation of photoplethysmography as a method to detect heart rate during rest and exercise. Journal of Medical Engineering and Technology, 39 (5), 264-271.

[15] Vandenberk, T., Stans, J., Mortelmans, C., Van Haelst, R., Van Schelvergem, G., Pelckmans, C., Smeets, C.J., Lanssens, D., De Canniere, H., Storms, V., Thijs, I.M., Veas, B., Vandervoort, P.M. (2017). Clinical validation of heart rate apps: Mixed-methods evaluation study. JMIR mHealth and uHealth, 5 (8), e129.

[16] Peng, R.C., Zhou, X.L., Lin, W.H., Zhang, Y.T. (2015). Extraction of heart rate variability from smartphone photoplethysmograms. Computational and Mathematical Methods in Medicine, 2015, 516826.

[17] Madan, C.R., Harrison, T., Mathewson, K.E. (2018). Noncontact measurement of emotional and physiological changes in heart rate from a webcam. Psychophysiology, 55 (4).

[18] Macwan, R., Benezeth, Y., Mansouri, A. (2018). Remote photoplethysmography with constrained ICA using periodicity and chrominance constraints. BioMedical Engineering OnLine, 17 (1), 22.

[19] Smith, S.W. (2003). Digital Signal Processing: A Practical Guide for Engineers and Scientists. $1^{\text {st }}$ Edition. Newnes.

[20] Hejjel, L., Rőth, E. (2004). What is the adequate sampling interval of the ECG signal for heart rate variability analysis in the time domain? Physiological Measurement, 25, 1405-1411.

[21] García-González, M.A., Fernández-Chimeno, M., Ramos-Castro, J. (2004). Bias and uncertainty in heart rate variability spectral indices due to the finite ECG sampling frequency. Physiological Measurement, 25 (2), 489-504.
[22] Singh, B., Singh, M., Banga, V.K. (2014). Sample entropy based HRV: Effect of ECG sampling frequency. Biomedical Science and Engineering, 2 (3), 68-72.

[23] Ziemssen, T., Gasch, J., Ruediger, H. (2008). Influence of ECG sampling frequency on spectral analysis of RR intervals and baroreflex sensitivity using the EUROBAVAR data set. Journal of Clinical Monitoring and Computing, 22 (2), 159-68.

[24] Daskalov, I.K., Christov, I.I. (1997). Improvement of resolution in measurement of electrocardiogram RR intervals by interpolation. Medical Engineering and Physics, 19, 375-379.

[25] Baek, H.J., Shin, J., Jin, G., Cho, J. (2017). Reliability of the parabola approximation method in heart rate variability analysis using low-sampling-rate photoplethysmography. Journal of Medical Systems, 41 (12), 189.

[26] Mahdiani, S., Jeyhani, V., Peltokangas, M., Vehkaoja, A. (2015). Is $50 \mathrm{~Hz}$ high enough ECG sampling frequency for accurate HRV analysis? In $37^{\text {th }}$ Annual International Conference of the IEEE Engineering in Medicine and Biology Society (EMBC), 25-29 August 2015, Milan, Italy. IEEE, 5948-5951.

[27] Choi, A., Shin, H. (2017). Photoplethysmography sampling frequency: Pilot assessment of how low can we go to analyze pulse rate variability with reliability? Physiological Measurement, 38 (3), 586-600.

[28] Task Force of the European Society of Cardiology and the North American Society of Pacing and Electrophysiology. (1996). Heart rate variability. Standards of measurement, physiological interpretation, and clinical use. Circulation, 93, 10431065.

[29] Elgendi, M. (2012). On the analysis of fingertip photoplethysmogram signals. Current Cardiology Reviews, 8 (1), 14-25.

[30] Shannon, C.E. (1948). A mathematical theory of communication. Bell System Technical Journal, 27 (3), 379-423.

[31] Shannon, C.E. (1949). Communications in the presence of noise. Proceedings of IRE, 37, 10-21.

Received February 20, 2019

Accepted August 30, 2019 